\title{
Human papillomavirus type 16 oncoprotein E7 suppresses cadherin-mediated cell adhesion via ERK and AP-1 signaling
}

\author{
HONG YUAN $^{1}$, SATOKO ITO ${ }^{1}$, TAKESHI SENGA ${ }^{1}$, TOSHINORI HYODO ${ }^{1}$, TOHRU KIYONO ${ }^{3}$, \\ FUMITAKA KIKKAWA ${ }^{2}$ and MICHINARI HAMAGUCHI ${ }^{1}$ \\ ${ }^{1}$ Division of Cancer Biology, ${ }^{2}$ Depertment of Obstetrics and Gynecology, Nagoya University Graduate School of Medicine, \\ 65 Tsurumai-cho, Showa-ku, Nagoya 466-8550; ${ }^{3}$ Virology Division, National Cancer Center \\ Research Institute, 5-1-1 Tsukiji, Chuo-ku, Tokyo 104-0045, Japan
}

Received January 19, 2009; Accepted March 18, 2009

DOI: 10.3892/ijo_00000341

\begin{abstract}
Human papillomaviruses (HPV) are the main etiological factor for cervical carcinoma. HPV-16 is the most prevalent high-risk HPV-genotype found in HPV-associated cancers. We studied the effect of HPV-16 E7 oncoprotein on cadherin-mediated cell adhesion. The expression of E7 strongly suppressed the cadherin-mediated cell adhesion in the rat fibroblast cell line $3 \mathrm{Y} 1$. This suppression was associated with the decreased expression of $\mathrm{N}$-cadherin at the transcriptional level. The treatment of $3 \mathrm{Y} 1$ cells that express E7 (E7-3Y1) with MEK inhibitor recovered the cadherinmediated cell adhesion together with the accumulation of $\mathrm{N}$ cadherin at the cell-cell contact site. Moreover, the suppression of c-Jun, which is the element of AP-1 transcriptional factor, leads to the recovery of N-cadherin expression and cadherin-mediated cell adhesion in E7-3Y1 cells. Taken together, our results demonstrate that E7 regulates cadherin-mediated cell adhesion through the modulation of cadherin expression via the MEK-ERK and AP-1 signaling pathway.
\end{abstract}

\section{Introduction}

Human papillomaviruses (HPV) are recognized as the most important causal agents in cervical carcinoma development. Although there are approximately $40 \mathrm{HPV}$ genotypes that infect the genital mucosa, HPV-16 is the most frequent viral type and associated with about a half of all cervical carcinoma (1). Two viral oncoproteins of HPV-16, E6 and E7, are essential factors for HPV-induced cellular transformation. The major functions of E6 and E7 oncoproteins in infected cells are inactivation of p53 and retinoblastoma protein $(\mathrm{pRb})(2,3)$. In addition to inhibitory effect on tumor

Correspondence to: Dr Michinari Hamaguchi, Division of Cancer Biology, Nagoya University Graduate School of Medicine, 65 Tsurumai-cho, Showa-ku, Nagoya 466-8550, Japan

E-mail: mhamagu@med.nagoya-u.ac.jp

Key words: human papillomavirus type 16, HPV-16 oncoprotein E7, $\mathrm{N}$-cadherin, cell to cell adhesion, ERK, AP-1 suppressor proteins, E6 and E7 oncoproteins have other target proteins and affect tumorigenesis in a wide variety of aspects (4-6).

One of the most outstanding properties of tumor cells is metastasis. The metastatic process requires tumor cells to detach and migrate away from the primary tumor, intravasate into blood or lymphatic vessels, and re-attach to metastasizing sites. Cadherin/catenin complex is the most important molecular structure of cell-cell adhesion $(7,8)$, and during first step of metastasis, disruption of cadherin mediated cellcell adhesion occurs. Among over 40 cadherin superfamily members, the classic cadherins, such as E-, P-, and N-cadherin, are transmembrane glycoproteins whose extracellular domain binds to similar cadherins of adjacent cells in a calciumdependent manner (9). In tumor cells, the system of cadherinmediated cell-cell adhesion is often disrupted because of suppression of cadherin expression, abnormality of cadherin distribution, phosphorylation of cadherin, or expression of different types of cadherin (10-12). Consequently, tumor cells dissociate from primary tumor and take the step to metastasis.

A previous study reported that expression of HPV-16 E7 alone is sufficient for focal transformation of an immortalized rat fibroblast cell line 3 Y1 (13). To obtain more clues about cell transformation by HPV-16 E7, especially from the point of cell-cell adhesion, we examined cadherin expression and cadherin-dependent cell aggregation ability using 3Y1 cells that constitutively express E7 (E7-3Y1). In this report, we show that E7 expression induces the suppression of $\mathrm{N}$-cadherin expression at the transcriptional level, and reduces cell-cell adhesion. This phenomenon is recovered by inhibition of ERK1/2 activity. Moreover, the suppression of c-Jun, which is one of the components of AP-1 transcription complex, by treatment with siRNA causes the recovery of $\mathrm{N}$-cadherin expression and cadherin-mediated cell adhesion in E7-3Y1 cells.

\section{Materials and methods}

Antibodies and chemicals. Primary antibodies used for immunoblotting were purchased from the following manufacturers: anti-E7 antibody (Zymed); anti-N-cadherin and 
anti-c-Jun antibodies (Transduction Laboratories); anti-ß-actin antibody (Sigma); anti-phospho-ERK1/2 (Thr202/Tyr204) antibody (Cell Signaling Technology); anti-ERK2 antibody (Santa Cruz). Secondary antibodies used for immunoblotting were anti-mouse $\mathrm{IgG}$ conjugated to horseradish peroxidase (Biosource) and Protein A conjugated to horseradish peroxidase (GE Healthcare). Primary antibody used for immunofluorescence staining was anti-N-cadherin antibody (Transduction Laboratories). Secondary antibody used for immunofluorescence staining was anti-mouse IgG conjugated to Alexa Fluor 488 (Invitrogen). MEK1/2 inhibitor U0126 was purchased from Cell Signaling Technology. Cells were treated with U0126 $10 \mu \mathrm{M} 24 \mathrm{~h}$ before assays.

Cell culture. 3 Y1 cells and E7-3Y1 cells were maintained in Dulbecco's-modified Eagle's medium (DMEM) supplemented with $10 \%$ fetal bovine serum (FBS).

Immunoblotting. Cells were lysed with Laemmli sample buffer (20\% glycerol, 135 mM Tris- $\mathrm{HCl}$ pH 6.8, 4\% SDS, 10\% 2mercaptoethanol, $0.003 \%$ BPB) and boiled $5 \mathrm{~min}$. Protein concentrations of lysates were measured using RC-DC Protein Assay (Bio-Rad). Equal protein quantities were loaded onto SDS-polyacrylamid electrophoresis (SDS-PAGE) gels, and transferred to PVDF membrane (Millipore). The membrane was blocked with $5 \%$ non-fat skimmed milk and incubated with the each primary antibody for $1 \mathrm{~h}$, washed with TBS-T buffer (100 mM Tris- $\mathrm{HCl} \mathrm{pH} 7.4,9 \% \mathrm{NaCl}, 0.5 \%$ Tween-20) and then incubated with the secondary antibodies. Proteins were visualized by enhanced chemiluminescence (GE Healthcare).

Reverse transcription-PCR. Total RNA was purified from cultured cells and cDNA was synthesized from $5 \mu \mathrm{g}$ of total RNA using ReverTra Ace (Toyobo). The oligonucleotide primers used for RT-PCR were as follows: N-cadherin: sense (5'-GGACAGTTCCTGAGGGATCA-3'); antisense (5'-TGG TTTGACCACGGTGACTA-3'). GAPDH: sense (5'-ACCAC AGTCCATGCCATCAC-3'); antisense (5'-TCCACCACCCT GTTGCTGTA-3'). RT-PCR products were analyzed on agarose gels containing $0.4 \mu \mathrm{g} / \mathrm{ml}$ ethidium bromide.

Immunofluorescence staining. Cells were fixed with $4 \%$ paraformaldehyde, permealized with $0.5 \%$ Triton X-100 for $5 \mathrm{~min}$, blocked with 7\% fetal bovine serum in PBS for $30 \mathrm{~min}$, and incubated with the indicated primary antibody followed by the secondary antibody.

Cell aggregation assay. Cadherin-mediated cell aggregation was assayed as described previously (14). Briefly, cells were treated with $0.01 \%$ crystallized trypsin in $\mathrm{Ca}^{2+}$ - and $\mathrm{Mg}^{2+}$-free HEPES buffered saline (HCMF, pH 7.4) or HCMF with $1 \mathrm{mM}$ $\mathrm{CaCl}_{2}$ (HMF) at $37^{\circ} \mathrm{C}$ for $20 \mathrm{~min}$ and then washed with HCMF to obtain single cell suspensions. The cells were then washed with $0.05 \%$ soybean trypsin inhibitor in HCMF and incubated at $37^{\circ} \mathrm{C}$ for 15 min with gyratory shaking in HMF with $1 \%$ bovine serum albumin. The data were plotted as the percentage of single cells relative to total single cells at zero time, and are presented as the average of determination from three different experiments.

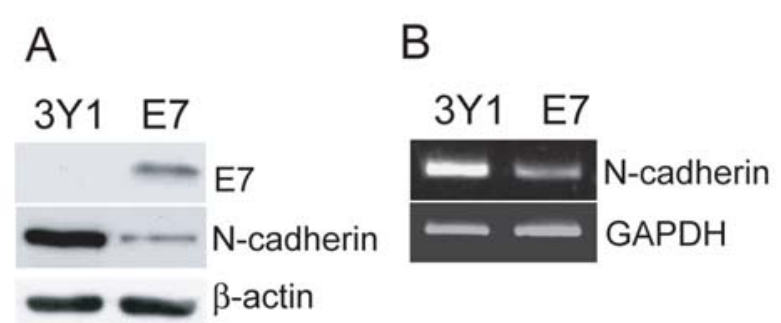

C

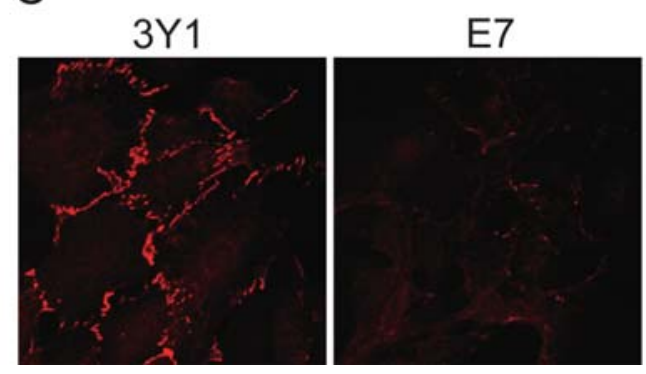

Figure 1. Decreased expression of N-cadherin by E7. (A) Expression of E7 and $\mathrm{N}$-cadherin in $3 \mathrm{Y} 1$ and E7-3Y1 (E7) cells was examined by immunoblotting. B-actin was used as a loading control. (B) mRNA expression of $\mathrm{N}$-cadherin in 3 Y 1 and E7-3Y1 cells was examined by RT-PCR. GAPDH was used as a control. (C) Expression and localization of N-cadherin in 3Y1 and E7-3Y1 cells was confirmed by immunofluorescence staining with anti-N-cadherin antibody.

siRNA and its transfection. siRNA against c-Jun was designed and synthesized by Invitrogen. The sequence of siRNA is 5'-AACUGCUGCGUUAGCAUGAGUUGGC-3'. The scrambled siRNA (Invitrogen) was used as a control. siRNA $(20 \mathrm{nM})$ was transfected to cells using Lipofectamine 2000 (Invitrogen) according to manufacturer's protocol. Fortyeight hours after the siRNA transfection, cells were used for the indicated assays.

\section{Results}

Expression of $\mathrm{N}$-cadherin and cadherin-mediated cell-cell adhesion in $3 Y 1$ cells and E7-3Y1 cells. To investigate the effects of E7 expression on cell-cell adhesion, we established cell lines that constitutively express E7 in 3Y1 cells (E7-3Y1). Because the major cadherin that is expressed in $3 \mathrm{Y} 1$ cells was $\mathrm{N}$-cadherin (15), we examined $\mathrm{N}$-cadherin expression in 3 Y1 cells and E7-3Y1 cells by immunoblotting, and found that the expression of N-cadherin in E7-3Y1 cells was suppressed compared with that of 3 Y 1 cells (Fig. 1A). To ascertain whether the suppression of $\mathrm{N}$-cadherin expression was at the transcriptional level, we checked mRNA expression of N-cadherin using RT-PCR. In E7-3Y1 cells, mRNA expression of $\mathrm{N}$-cadherin was decreased compared with that of 3 Y 1 cells (Fig. 1B). Next, we performed immunostaining to observe the localization of $\mathrm{N}$-cadherin in cells. As shown in Fig. 1C, 3 Y 1 cells formed tight association between cells and $\mathrm{N}$-cadherin accumulated as a bundle-like structure at cell-cell contact sites. In contrast, E7-3Y1 cells lost the tight adhesion, and the accumulation of $\mathrm{N}$-cadherin to the cell-cell contact sites was scarcely observed. To compare the 

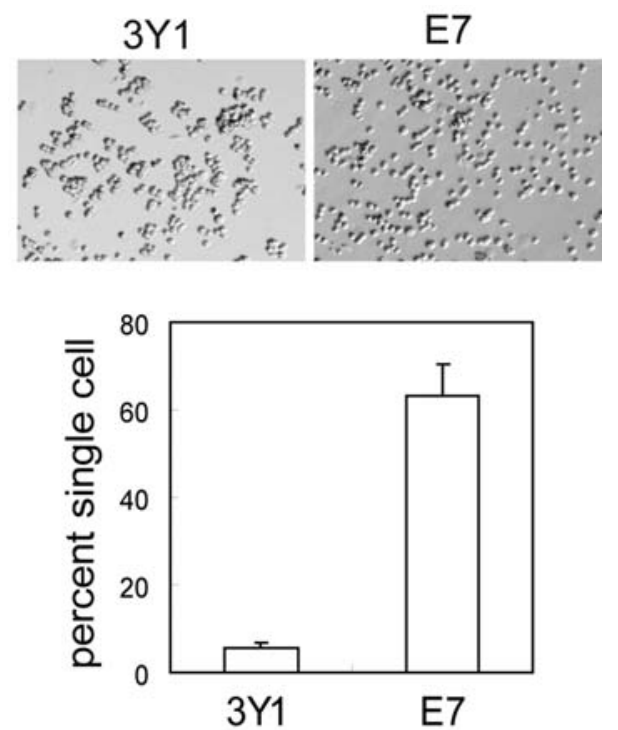

Figure 2. Suppression of cell aggregation by E7. Cell aggregation assay of $3 \mathrm{Y} 1$ and E7-3Y 1 cells was performed as described in Materials and methods. Representative images of cell aggregation assay are shown. Percentages of single cells were counted and the mean averages \pm SD from three independent experiments are indicated.

cadherin mediated adhesive properties between $3 \mathrm{Y} 1$ and E7$3 \mathrm{Y} 1$ cells, cells were dissociated by trypsin in the presence of $\mathrm{CaCl}_{2}$ and then shaken to allow aggregation. As shown in Fig. 2, the aggregation of cells observed in $3 \mathrm{Y} 1$ cells was strongly inhibited in E7-3Y1 cells. These results suggest that cadherin expression and cadherin-mediated cell-cell adhesion is strongly suppressed in E7-3Y1 cells.

Suppression of ERK1/2 activity restores $N$-cadherin expression and cadherin-mediated cell-cell adhesion in E7-3Y1 cells. Previous studies have reported that MAP kinases were activated in clinical samples and cell lines of HPV-16 positive cervical cancer $(16,17)$. Therefore, we checked the activity of ERK1/2 by immunoblotting with anti-phosphoERK1/2 antibody, and found that the phosphorylation of ERK1/2 was significantly increased in E7-3Y1 cells (Fig. 3A). We next examined whether $\mathrm{N}$-cadherin expression was recovered by the inhibition of ERK1/2 activity. Treatment of E7-3Y1 cells with MEK1/2 inhibitor, U0126, suppressed the phosphorylation level of ERK1/2, and restored the expression of N-cadherin (Fig. 3A). Similarly, mRNA expression of $\mathrm{N}$-cadherin was recovered in E7-3Y1 cells treated with U0126 (Fig. 3B), suggesting that the expression of $\mathrm{N}$-cadherin is controlled at the transcriptional level in an ERK1/2 signaling-dependent manner. Immunofluorescence staining of N-cadherin showed that the treatment of E7-3Y1 cells with U0126 recovered the morphology, cell-cell adhesion and accumulation of $\mathrm{N}$-cadherin at cell-cell adhesion sites (Fig. 3C). Furthermore, cadherin-mediated cell aggregation was restored in E7-3Y1 cells by U0126 treatment (Fig. 3D). These results indicate that ERK pathway is indispensable for the suppression of cadherin-mediated cell-cell adhesion in E7-3Y1 cells.
A

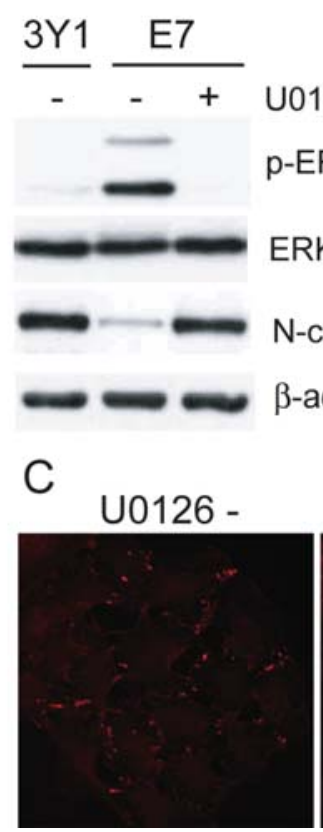

B

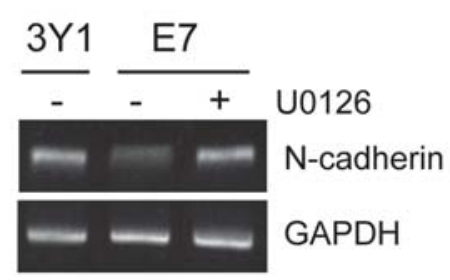

D
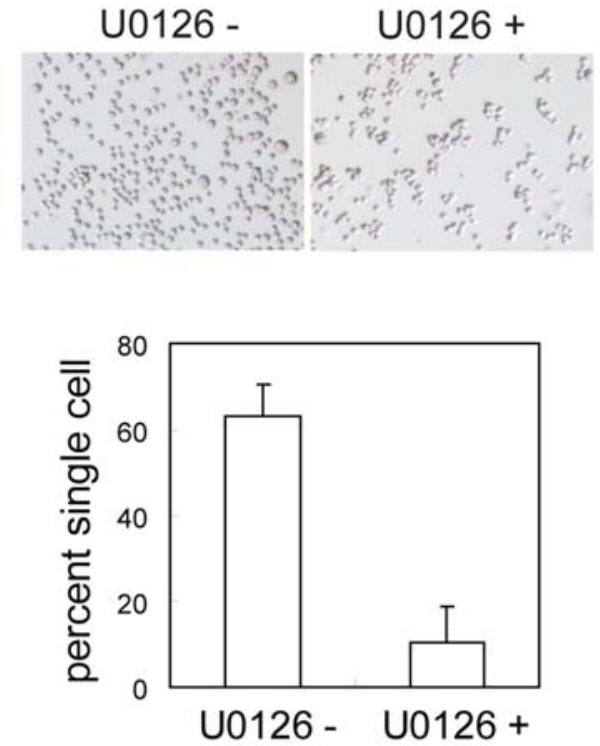

Figure 3. MEK inhibitor restores N-cadherin expression and cell aggregation. (A) Phosphorylation of ERK-1/2 and expression of N-cadherin in 3Y1 and E7-3Y1 cells treated with or without U0126 were examined by immunoblotting. B-actin was used as a loading control. (B) mRNA expression of N-cadherin in 3Y1 and E7-3Y1 cells treated with or without U0126 was examined by RT-PCR. GAPDH was used as a control. (C) Expression and localization of N-cadherin in E7-3Y1 cells with or without U0126 was confirmed by immunofluorescence staining with anti-N-cadherin antibody. (D) Cell aggregation assay of 3 Y1 and E7-3Y1 cells with or without U0126 was performed as described in Materials and methods. Percentages of single cells were counted and the mean averages \pm SD from three independent experiments are indicated. 
A

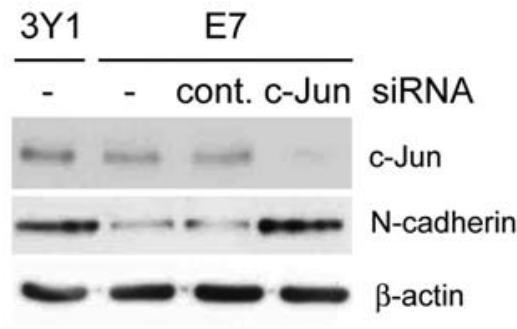

B

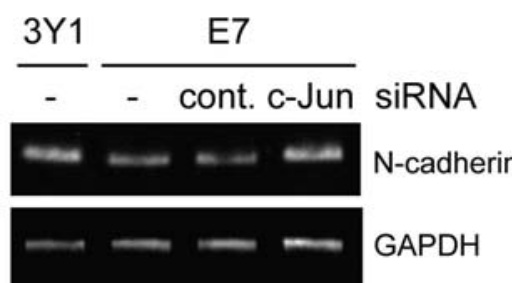

Figure 4. Silencing of c-Jun by siRNA restored expression of N-cadherin. (A) E7-3Y1 cells were transfected with control (cont.) or c-Jun siRNA. Expression of c-Jun and $\mathrm{N}$-cadherin was examined by immunoblotting. B-actin was used as a loading control. (B) mRNA expression of N-cadherin in control or c-Jun siRNA treated cells was examined by RT-PCR. GAPDH was used as a control.
c-Jun siRNA treatment restores $N$-cadherin expression and cadherin-mediated cell-cell adhesion in E7-3Y1 cells. To confirm the requirement of AP-1 signaling, one of the downstream transcriptional factor of ERK, we next examined the effect of c-Jun siRNA on E7-3Y1 cells. c-Jun interacts with Fos as well as other Jun proteins to generate a dimer called the AP-1 transcriptional complex. Forty-eight hours after the transfection of control or c-Jun siRNA to E7-3Y1 cells, cells were lysed and subjected to immunoblotting. Knockdown of c-Jun markedly increased $\mathrm{N}$-cadherin expression in E7-3Y1 cells (Fig. 4A), and the recovery of N-cadherin mRNA expression was also observed (Fig. 4B). We next examined the expression and localization of N-cadherin by immunofluorescence staining. Twenty-four hours after c-Jun siRNA transfection, E7-3Y1 cells were plated on coverslips and cultured for another $24 \mathrm{~h}$. Cells were then fixed and stained with antibodies to c-Jun, N-cadherin and DAPI for nuclear staining. As shown in Fig. 5A, we could not observe any expression of c-Jun in some cells (arrowheads), indicating that c-Jun siRNA was effective. N-cadherin accumulation at cell-cell contact site was restored in c-Jun-knockdown cells. In contrast, $\mathrm{N}$-cadherin was not observed at cell membrane in c-jun expressing cells (asterisks). Furthermore, cadherinmediated cell aggregation was restored in E7-3Y1 cells by
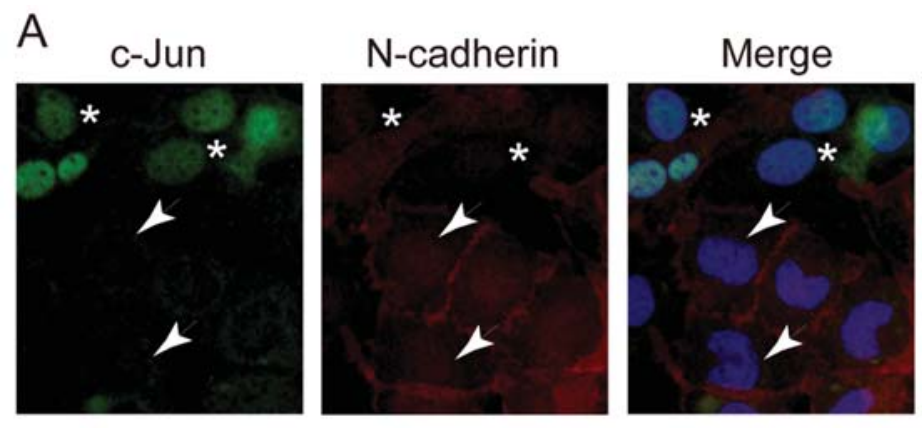

B

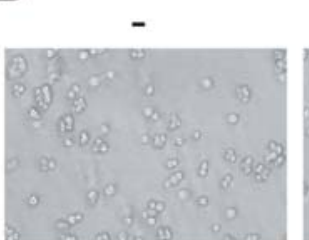

cont. siRNA
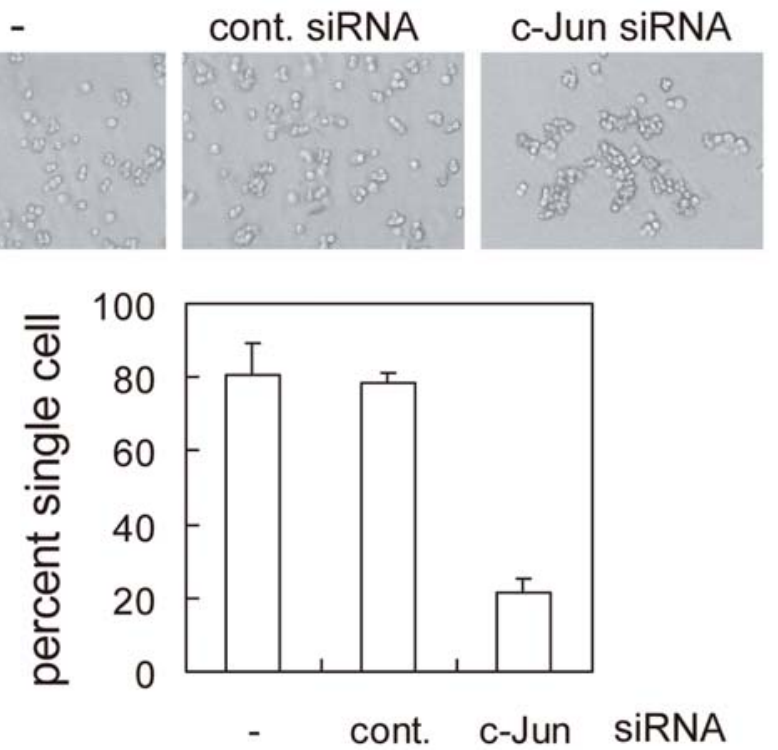

Figure 5. Localization of N-cadherin and cell aggregation by c-Jun siRNA. (A) Expression of c-Jun (green) and N-cadherin (red) in E7-3Y1 cells treated with c-Jun siRNA was confirmed by immunofluorescence staining with anti-c-Jun and anti-N-cadherin antibodies. Nuclei were costained with DAPI (blue) in Merge. Arrowheads indicate cells with reduced expression of c-Jun and asterisks indicate c-Jun expressing cells. (B) Cell aggregation assay of E7-3Y1 cells with or without siRNA was performed as described in Materials and methods. Percentages of single cells were counted and the mean averages \pm SD from three independent experiments are indicated. 
c-Jun siRNA treatment (Fig. 5B). These results suggest that AP-1 signaling is involved in suppression of N-cadherin expression and cadherin-mediated cell-cell adhesion in E7-3Y1 cells.

\section{Discussion}

In this report, we show that HPV-16 E7 causes the suppression of cadherin-mediated cell adhesion. This suppression is caused by the reduced expression of cadherin at the transcriptional level in both ERK and AP-1 signal-dependent manner. It has been reported that HPV-16 E7 oncoprotein interacts with several cellular proteins. Among these, proteins of $\mathrm{pRB}$ family are the main targets of E7 (3). The binding of E7 and $\mathrm{pRb}$ causes the disruption of pRb-E2F complexes, leading to the activation of E2F mediated transcription of particular genes involved in DNA synthesis and cell-cycle progression (18). Many other E7 binding proteins are also related to cell cycle and cell proliferation (4). Therefore, the analysis of tumorigenesis caused by E7 has been performed mostly from the view of the deregulation of cell cycle and cell proliferation. However, in tumor progression, especially in invasion and metastasis, disruption of cell to cell adhesion plays a pivotal role. Cadherins are one of the major molecules that regulate cell to cell adhesions between adjacent cells, and loss or reduction of cadherin-mediated cell adhesion is related to invasion and metastasis of a wide variety of tumors. (10). Cadherins also associate with various signaling molecules, and the decrease of cadherin expression not only causes disruption of cell to cell adhesion but also induces aberrant multiple signaling pathways that are involved in tumorigenesis $(19,20)$. Recent study showed that silencing of E7 in HPV16-transformed keratinocytes recovered E-cadherin expression, indicating a possible role of E-cadherin regulation for E7-mediated tumorigensis (21). Our results suggest that down-regulation of cadherin expression is one of the critical pathways for invasion and metastasis of HPVinduced tumors.

The Ras/ERK pathway plays a critical role in tumorigenesis (22), and E7 expression in 3 Y 1 cells caused activation of this pathway. ERK signal activates members of AP-1 family protein such as c-Jun and c-Fos, which regulate expression of genes involved in cell proliferation, differentiation, malignant transformation and metastasis (23). In this study we showed that suppression of ERK1/2 activity by MEK1/2 inhibitor and silencing of c-Jun by siRNA restored the expression of $\mathrm{N}$-cadherin. These results indicate that ERK and AP-1 pathway plays a critical role in the regulation of $\mathrm{N}$-cadherin expression. Analysis of promoter region of chicken $\mathrm{N}$-cadherin showed that there were some AP-1 binding sequences, however, whether AP-1 directly regulates $\mathrm{N}$-cadherin expression is still unknown (24). Silencing of E-cadherin expression is mediated by the action of transcriptional factors such as Slug, Snail and Twist. It is possible that N-cadherin down-regulation by ERK and AP-1 pathway in E7-transformed 3 Y 1 cells are also regulated by other transcriptional factors whose expression are regulated by AP-1 activity. We need further studies to clarify the mechanism of how N-cadherin expression is regulated by E7-mediated ERK and AP-1 activation.
In conclusion, we have demonstrated that HPV-16 E7 suppressed cadherin expression at the transcriptional level and cadherin-mediated cell adhesion in 3Y1. In addition, inhibition of ERK-1/2-AP-1 signal pathway blocks the suppression of cadherin expression and restores cadherinmediated cell adhesion. Our results indicate a possible new mechanism of E7-mediated tumorigenesis that is dependent on the suppression of cadherin expression.

\section{Acknowledgments}

We thank members of the Hamaguchi Laboratory for their technical assistance and helpful discussion. This study was supported by a Grant-in-Aid for COE research from the Ministry of Education, Science, Culture and Technology of Japan.

\section{References}

1. Munoz N, Bosch FX, De Sanjose S, Herrero R, Castellsague X, Shah KV, Snijders PJF and Meijer CJLM: Epidemiologic classification of human papillomavirus types associated with cervical cancer. N Engl J Med 348: 518-527, 2003.

2. Werness BA, Levine AJ and Howley PM: Association of human papillomavirus type 16 and 18 E6 proteins with p53. Science 248: 76-79, 1990.

3. Dyson N, Howley PM, Munger K and Harlow E: The human papillomavirus-16 E7 oncoprotein is able to bind to the retinoblastoma gene product. Science 243: 934-937, 1989.

4. Narisawa-Saito $M$ and Kiyono T: Basic mechanisms of highrisk human papillomavirus-induced carcinogenesis: role of E6 and E7 proteins. Cancer Sci 98: 1505-1511, 2007.

5. Motoyama S, Ladines-Llave CA, Villanueva SL and Maruo T: The role of human papilloma virus in the molecular biology of cervical carcinogenesis. Kobe J Med Sci 50: 9-19, 2004.

6. Munger K, Baldwin A, Edwards KM, Hayakawa H, Nguyen CL, Owens M, Grace M and Huh KW: Mechanisms of human papillomavirus-induced oncogenesis. J Virol 78: 11451-11460, 2004.

7. Takeichi M: Cadherin cell adhesion receptors as a morphogenetic regulator. Science 251: 1451-1455, 1991.

8. Gumbiner BM: Regulation of cadherin adhesive activity. J Cell Biol 148: 399-403, 2000

9. Halbleib JM and Nelson WJ: Cadherins in development: cell adhesion, sorting, and tissue morphogenesis. Genes Dev 20: 3199-3214, 2006.

10. Takeichi M: Cadherins in cancer: implications for invasion and metastasis. Curr Opin Cell Biol 5: 806-811, 1993.

11. Matsuyoshi N, Hamaguchi M, Taniguchi S, Nagafuchi A, Tsukita S and Takeichi M: Cadherin-mediated cell-cell adhesion is perturbed by $v$-src tyrosine phosphorylation in metastatic fibroblasts. J Cell Biol 118: 703-714, 1992.

12. Wheelock MJ, Shintani Y, Maeda M, Fukumoto Y and Johnson KR: Cadherin switching. J Cell Sci 121: 727-735, 2008.

13. Watanabe S, Sato H, Komiyama N, Kanda T and Yoshiike K: The E7 functions of human papillomaviruses in rat $3 \mathrm{Y} 1$ cells. Virology 187: 107-114, 1992.

14. Hamaguchi M, Matsuyoshi N, Ohnishi Y, Gotoh B, Takeichi M and Nagai $\mathrm{Y}$ : p60 $60^{v-s r c}$ causes tyrosine phosphorylation and inactivation of the $\mathrm{N}$-cadherin-catenin cell adhesion system. EMBO J 12: 307-314, 1993.

15. Itano N, Atsumi F, Sawai T, Yamada Y, Miyaishi O, Senga T, Hamaguchi $\mathrm{M}$ and Kimata $\mathrm{K}$ : Abnormal accumulation of hyaluronan matrix diminishes contact inhibition of cell growth and promotes cell migration. Proc Natl Acad Sci USA 99: 3609-3614, 2002.

16. Perez-Plasencia C, Vazquez-Oritz G, Lopez-Romero R, PinaSanchez P, Moreno J and Salcedo M: Genome wide expression analysis in HPV 16 cervical cancer: identification of altered metabolic pathways. Infect Agent Cancer 2: 16-27, 2007.

17. Subbaramaiah K and Dannenberg AJ: Cyclooxygenase-2 transcription is regulated by human papillomavirus $16 \mathrm{E} 6$ and E7 oncoproteins: evidence of a corepressor/coactivator exchange. Cancer Res 67: 3976-3985, 2007. 
18. Dyson N: The regulation of E2F by pRB-family proteins. Genes Dev 12: 2245-2265, 1998.

19. Onder TT, Gupta PB, Mani SA, Yang J, Lander ES and Weinberg RA: Loss of E-cadherin promotes metastasis via multiple downstream transcriptional pathways. Cancer Res 68: 3645-3654, 2008

20. Arima $Y$, Inoue $Y$, Shibata $T$, Hayashi $H$, Nagano O, Saya $H$ and Taya $\mathrm{Y}: \mathrm{Rb}$ depletion results in degradation of E-cadherin and induction of cellular phenotypic change that are characteristic of the epithelial-to-mesenchymal transition. Cancer Res 68: 5104-5112, 2008.

21. Caberg JHD, Hubert PM, Begon DY, Herfs MF, Roncarati PJ, Boniver JJ and Delvenne PO: Silencing of E7 oncogene restores functional E-cadherin expression in human papillomavirus 16transformed keratinocytes. Carcinogenesis 29: 1441-1447, 2008.
22. McCubrey JA, Steelman LS, Chappell WH, Abrams SL, Wong EWT, Chang F, Lehmann B, Terrian DM, Milella M, Tafuri A, Stivala F, Libra M, Basecke J, Evangelisti C, Martelli AM and Franklin RA: Role of the Raf/MEK/ERK pathway in cell growth, malignant transformation and drug resistance. Biochim Biophys Acta 1773: 1263-1284, 2007.

23. Eferl R and Wagner EF: AP-1: a double-edged sword in tumorigenesis. Nat Rev Cancer 3: 859-868, 2003.

24. Li B, Paradies NE and Brackenbury RW: Isolation and characterization of the promoter region of the chicken $N$-cadherin gene. Gene 191: 7-13, 1997. 